\title{
De la peau de bête au smartphone
}

\section{Jean Martin}

Dr méd., membre de la rédaction

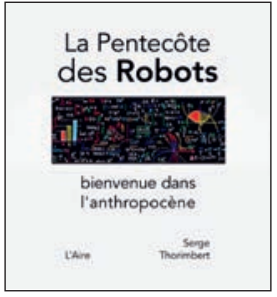

1 «Du fond des âges, l'outil est au service de la main; aujourd'hui, la main est à la traîne du 'device' électronique. Il n'y a plus de lien gratifiant pour l'esprit venu de ce que la main accomplit en guidant l'outil.»
Serge Thorimbert

La Pentecôte des Robots

Bienvenue dans l'anthropocène

Vevey: Editions de l'Aire, 2018, 240 p.

ISBN 9782940586813

Serge Thorimbert est un ingénieur de 59 ans, chef d'entreprise dans le domaine de l'énergie et des télécommunications, qui cultive aussi des préoccupations d'ordre humaniste et spirituel. Il vient de publier un livre sur l'avenir de nos rapports avec les développements technologiques. Une notion qu'il met en évidence est l'interfaçage entre l'humain et la nature: à savoir le fait que, depuis des millénaires, la progression de l'homme s'est faite en établissant/interposant des artefacts entre lui et l'environnement - depuis la peau de bête pour se vêtir jusqu'aux dispositifs sophistiqués en nombre croissant, smartphone, prothèses, etc. De manière telle à, aujourd'hui, "s'interfacer/se distancer de la nature au point que les équilibres de la vie sont rompus; or les équilibres de la biosphère sont indissociables de l'équilibre psychique collectif de nos sociétés.» Dans l'histoire de l'homme: "Avec l'émergence de la conscience, le corps et le psychisme humains ont peu à peu cessé de s'adapter à leur environnement. L'homme fait partie de la nature, en dépend mais entretient avec elle un rapport ambivalent qui lui vaut de dire l'aimer autant qu'il la détruit.»1

\section{Servitude numérique}

L'auteur insiste sur cette notion: "C'est un siphonnage permanent de nos cerveaux, de notre mémoire et de notre savoir-faire, qui s'opère par transfert vers le Cloud. Tout semble concourir pour chasser l'être humain (maillon faible et coûteux de la chaîne) en le remplaçant par des systèmes informatisés, voire par des robots.» L'hypothèse d'une perte de contrôle face à l'intelligence artificielle n'est plus un fantasme de roman d'anticipation. Des personnes comme Stephen Hawking, Bill Gates et Elon Musk l'envisagent.

«Nous sommes devenus démiurges en instaurant malgré nous une nouvelle 'divinité': omnisciente (accès à toutes les connaissances, Big Data, le Cloud), omniprésente (internet) et omnipotente (les moyens autonomes de calcul, de commande et de pilotage), dont les facultés nous dépassent.» Et: «Le technocentrisme ne propose aucun projet de société.» Ce qui caractérise les années récentes, c'est la prise de conscience qu'il n'y aura pas de retour en arrière. Toutefois: «Comment penser le futur alors que les composantes intriquées de notre présent sont illisibles? Et que ceux que nous créditons comme aptes à le faire ne s'expriment que de manière sectorielle, en experts au jargon pas pleinement appréhendable.» «Même lorsque le consensus est large, voire unanime, nous sommes incapables de prendre immédiatement les décisions qui s'imposent»une remarque qui en ce moment s'applique parfaitement aux questions de climat et de biodiversité.

\section{«Pas d'autre choix»}

Nous n'avons pas d'autre choix, dit Thorimbert, que d'embrasser une véritable maturité - dans notre appréciation de la situation et notre volonté d'effectuer les changements nécessaires. Mais la majorité de la population n'en est pas là, sensible qu'elle est aux discours alléguant qu'il n'y a pas de problème... Dans la foulée, «les pouvoirs dictatoriaux se renforcent pour préserver les privilèges des élites alors que les démocraties se fragilisent sous la montée en puissance des extrêmes». Des choses qu'on observe dans plusieurs pays.

\section{Une éthique des machines?}

«Il n'est pas inconcevable que les machines, dépourvues d'empathie mais aussi de cupidité et de goût du pouvoir, puissent accéder à une forme de 'conscience mathématique', froide mais déterminée à la préservation des équilibres de la vie sur la Terre...» Mécanisme qui nous protégerait de notre pulsion prométhéenne, de l'arrogance et de la mégalomanie que nous ne sommes pas en mesure de réfréner?! «La forme que prendrait l'action 'éthique' des machines est difficile à concevoir. Mais elles pourraient, mues par une forme de 'système vagal', couper les vivres de l'industrie de l'armement, de la production agrochimique, inhiber le fonctionnement de structures boursières et commerciales.» Vision pour le moins interpellante.

En guise de conclusion, cette citation de William Carlos Williams, médecin et poète américain (1883-1963): «L'homme a survécu jusqu'ici parce qu'il était trop ignorant pour réaliser ses désirs. Maintenant qu'il le peut, il doit les changer, ou périr.» 